\title{
Nitrogen as a spectroscopic tracer for measuring plasma flows in the high-field side SOL of ASDEX Upgrade
}

\section{Juuso Karhunen ${ }^{* a}$, Mathias Groth ${ }^{a}$, Petteri Heliste ${ }^{a}$, Toni Makkonen ${ }^{a}$, Antti Hakola ${ }^{b}$, Eleonora Viezzer ${ }^{c}$, Thomas Pütterich ${ }^{c}$ and the ASDEX Upgrade Team}

${ }^{a}$ Aalto University, Department of Applied Physics, Espoo, Finland

${ }^{b}$ VTT Technical Research Centre of Finland, Espoo, Finland

${ }^{c}$ Max-Planck-Institut für Plasmaphysik, Garching, Germany

E-mail: juuso.karhuneneaalto.fi

\begin{abstract}
New measurements of high-field side (HFS) scrape-off layer (SOL) flows were conducted in ASDEX Upgrade by Doppler spectroscopy of injected $\mathrm{N}$ impurities. $\mathrm{N}_{2}$ was injected into the plasma $13 \mathrm{~cm}$ above the HFS midplane during similar low-density L-mode discharges. The emission of $\mathrm{N}$ was observed both toroidally and poloidally at different radial locations by the HFS edge CXRS system. The flow velocities were determined from the Doppler shift of selected N(II) and N(III) lines within 460-465 nm. The results show migration of impurities towards the lower HFS divertor at the separatrix and away from it in the near SOL, the reversal being most probably driven by the perpendicular $\vec{E} \times \vec{B}$ drift. The results also suggest only partial equilibration of the $\mathrm{N}^{+}$ions with the background flow. Hence, the background D flow velocity cannot be directly determined from the measured $\mathrm{N}$ flows but has to be addressed by modelling of the injection experiment.
\end{abstract}

First EPs Conference on Plasma Diagnostics - 1st ECPD,

14-17 April 2015

Villa Mondragone, Frascati (Rome) Italy

\footnotetext{
* Speaker.
} 


\section{Introduction}

Measurements of plasma flow in the scrape-off layer (SOL) are needed for understanding material migration in the edge plasma. A specific region of interest is the high-field side (HFS), where the existing measurement data on flows is scarce. In ASDEX Upgrade, HFS SOL flows have earlier been studied by a reciprocating Langmuir probe close to the divertor entrance $[1,2]$ and by Doppler spectroscopy of injected C impurities around the HFS midplane region [3]. In Doppler spectroscopy, the flow velocity of the impurities is given by the Doppler shift of the spectral lines, while the line widths provide the impurity ion temperature. As only trace amounts of the impurity gas is injected into the plasma, it is the less intrusive of the two methods.

More recently, $\mathrm{N}$ has been used to replace $\mathrm{C}$ as a spectroscopic tracer in Doppler spectroscopy studies. $\mathrm{N}$ is already employed as a seeding gas in ASDEX Upgrade and various other tokamaks and is a more tolerated impurity, as it does not form similar co-deposited layers as C. Furthermore, simulations with the synthetic Doppler spectroscopy diagnostics [4] in the ERO code have shown similar radial impurity flow profiles with $\mathrm{N}_{2}$ and $\mathrm{CH}_{4}$ injections, suggesting equal applicability of $\mathrm{N}$ and $\mathrm{C}$ in flow measurements [5].

New flow measurements were performed at the HFS midplane of ASDEX Upgrade by Doppler spectroscopy in 2014 using $\mathrm{N}_{2}$ as the spectroscopic tracer. In these measurements, the experimental set-up used in [3] was extended with poloidal lines-of-sight (LOS), providing full characterization of the radial impurity flow profiles in both toroidal and poloidal directions. This way, also the contribution of the perpendicular $\vec{E} \times \vec{B}$ drift could be addressed, enabling indirect investigation of the radial electric field in the SOL.

\section{Spectroscopic flow measurements in ASDEX Upgrade}
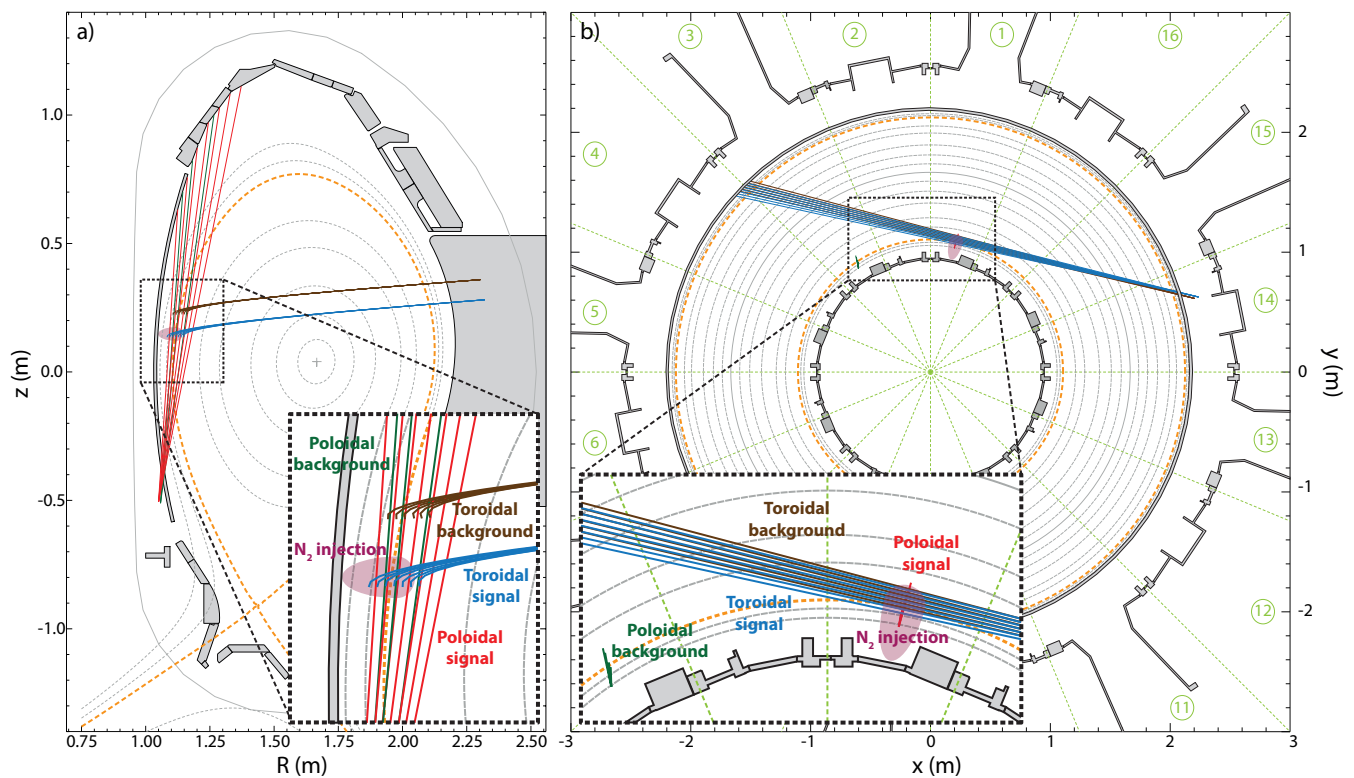

Figure 1: The geometry of the poloidal (a) and toroidal (b) spectroscopic lines-of-sight used for the flow measurements.

Flow measurements were performed parasitically during similar low-density L-mode discharges (AUG shot numbers \#31238-31250) with $I_{\mathrm{p}}=0.8 \mathrm{MA}, B_{\mathrm{t}}=-2.5 \mathrm{~T}, n_{\mathrm{e}}=1.9 \times 10^{19} \mathrm{~m}^{-3}$, 
auxiliary heating power of $P_{\mathrm{ECRH}}=1.3 \mathrm{MW}$ and the flat-top phase during $t=2.0-7.5 \mathrm{~s}$. The emission of the injected $\mathrm{N}$ was recorded in the wavelength range of 455-469 $\mathrm{nm}$ using the HFS edge CXRS system [6, 7]. The spectroscopic set-up, presented in Figure 1, consisted of seven toroidal and seven poloidal LOS viewing the injection region in different radial positions within $R=1.09$ $1.15 \mathrm{~m}$ and $R=1.10-1.18 \mathrm{~m}$, respectively, in the plane of the injection in toroidal sector 1 . In addition, a set of toroidal LOS approximately $10 \mathrm{~cm}$ higher and poloidal LOS in toroidal sector 3 were used to record the background emission, as shown in Figure 1. Undesirably, the geometric design of the main experiment had the plasma rather close to the central column with $R_{\text {sep }} \approx 1.11 \mathrm{~m}$ at the injection height, leading to a limited number of LOS in the SOL.

According to Figure 1a, the poloidal LOS cover almost the entire HFS SOL, while the toroidal LOS extend throughout the entire plasma and detect also the LFS SOL in two different locations, as shown in Figure 1b. Hence, the measurements are line integrated, which can cause ambiguities in localizing the detected emission in regions where low ionization states of $\mathrm{N}$ are rare. However, emission of the injected $\mathrm{N}$ in the HFS SOL is expected to dominate the emission recorded from elsewhere in the plasma.
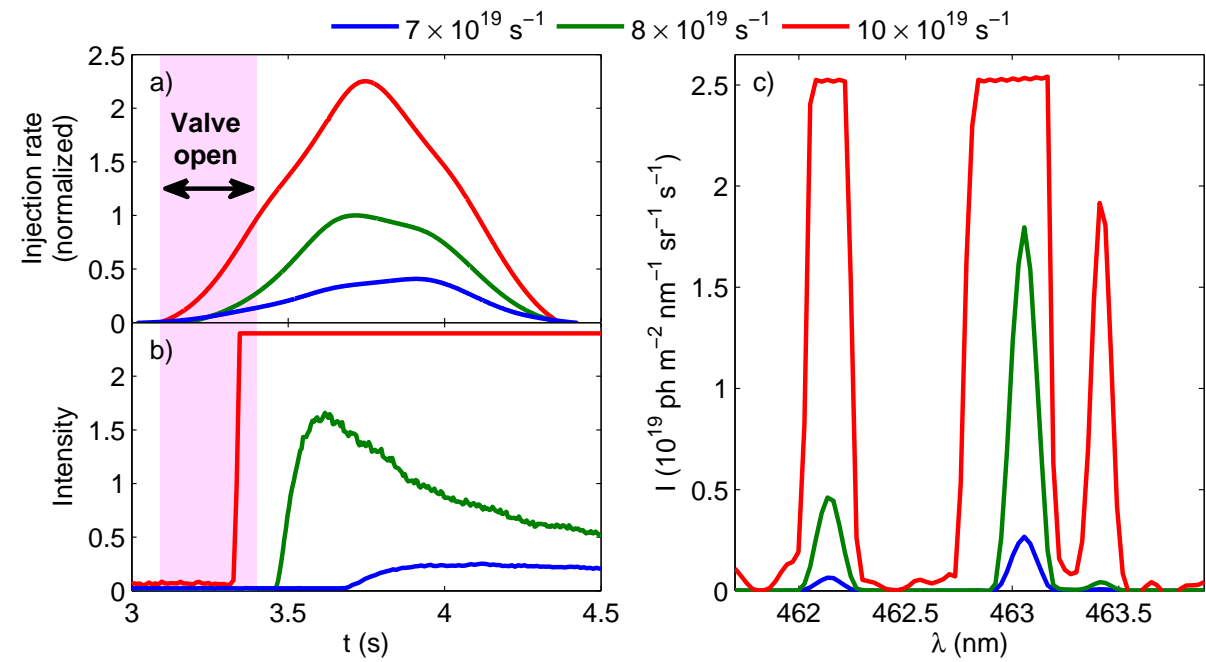

Figure 2: Temporal behaviour of a) the normalized $\mathrm{N}_{2}$ injection rate and b) the $\mathrm{N}$ (II) emission intensity, as well as c) part of the detected spectral lines for three different nominal injection rates. The light red area in (a) and (b) shows the open phase of the injection valve. The unit of intensity in (b) is the same as in (c).

$\mathrm{N}_{2}$ was injected into the plasma $13 \mathrm{~cm}$ above the HFS midplane during each discharge for $300-400 \mathrm{~ms}$ at nominal injection rates of 5.0-10.0 $\times 10^{19} \frac{1}{\mathrm{~s}}$ which were approximately an order of magnitude below the $\mathrm{D}$ fuelling rate. The injection rate was optimized such that a sufficiently intense signal was recorded without notably perturbing the plasma by the added impurity content. Figure 2 shows the temporal behaviour of the $\mathrm{N}_{2}$ injection rate, calculated using similar methods as in [8], and the emission intensity, as well as some of the detected spectral lines, during three discharges with different nominal injection rates. A highly non-linear piezo valve fed by a limiter reservoir was used, leading to the time lag in the injection in Figure 2a. With the lowest nominal rate of $5.0 \times 10^{19} \frac{1}{\mathrm{~s}}$, the valve did not open, and at $7.0 \times 10^{19} \frac{1}{\mathrm{~s}}$ the emission was weak, while at $10.0 \times 10^{19} \frac{1}{\mathrm{~s}}$, the amount of injected $\mathrm{N}$ caused saturation of the measured signal. An intense signal with no saturation was observed at $8.0 \times 10^{19} \frac{1}{\mathrm{~s}}$ which was selected for most of the discharges.

Due to uncalibrated measurement signal of the pressure drop in the $\mathrm{N}_{2}$ container, the actual injection rates could not be determined, but the normalized curves in Figure 2a show the proportions 
between the cases corresponding to different nominal injection rates. According to Figure 2a, the nominal injection rates do not correspond to the relations between the calculated ones, the low case reaching only half of the optimal injection and the highest one exceeding it by more than a factor of 2 . Figure $2 \mathrm{~b}$ also shows that the injection is more prompt at higher injection rates. However, this feature has no effect on the results in these studies.

\section{Results}

\subsection{Detection of nitrogen emission}

Altogether six N(II) lines and two N(III) lines, resulting from the transitions $2 s^{2} 2 p 3 p \rightarrow$ $2 s^{2} 3 p 3 s$ and $2 s^{2} 3 d \rightarrow 2 s^{2} 3 p$, respectively, were detected within 460-465 nm, as shown in Figure $3 \mathrm{a}-\mathrm{b}$. Emission was observed both before and during the injection, showing that also intrinsic, recycled $\mathrm{N}$ is detected. However, a 10-100-fold increase was observed in the intensity of the emission during the injection, suggesting that the injected $\mathrm{N}$ governs the emission.
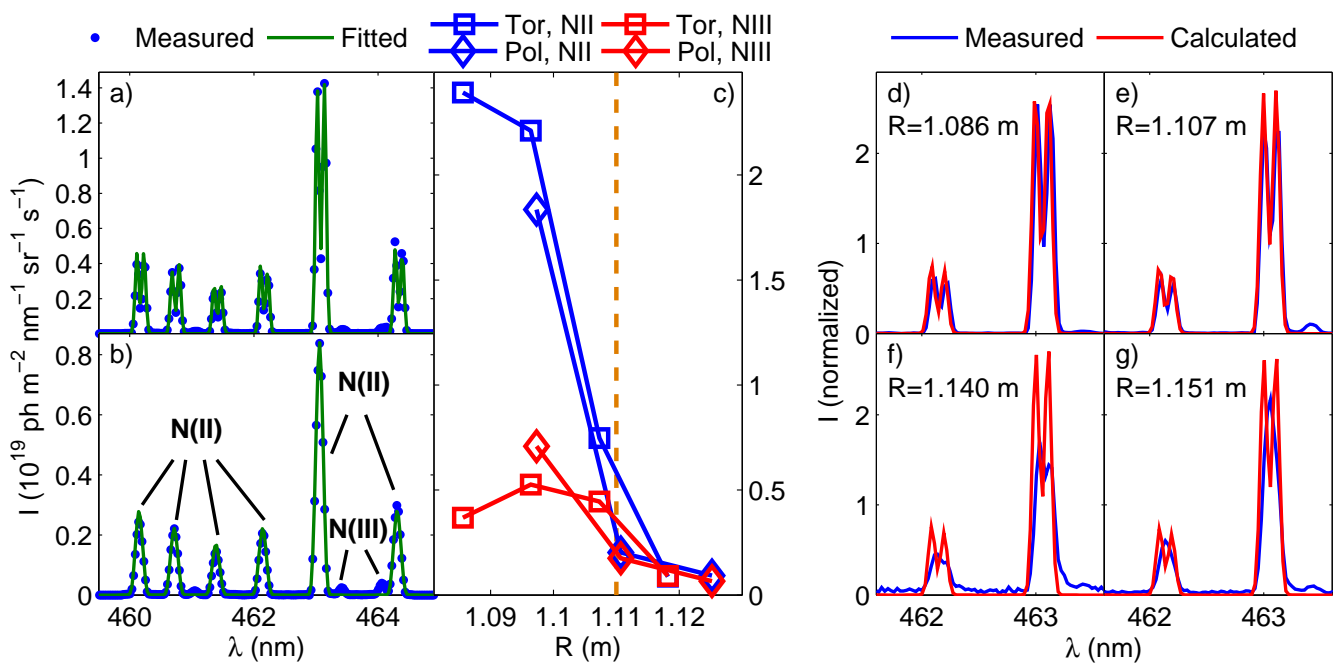

Figure 3: The detected $N($ II) and N(III) lines and the fits made to them in a) toroidal and b) poloidal direction, as well as radial emission profiles of $\mathrm{N}^{+}$an $\mathrm{N}^{2+}$ ions (c). The unit of intensity in (c) is the same as in (a) and (b), and the N(III) profiles have been scaled by a factor of 10 for comparability. The separatrix is represented by the orange dashed line. For the LOS in the SOL (d) and close to the separatrix (e), the line shapes predicted by Xpaschen under the magnetic conditions of the HFS midplane agree with the measured spectral lines, while a clear mismatch is observed inside the separatrix $(f, g)$.

Singly or doubly ionized $\mathrm{N}$ is not expected to exist inside the separatrix, which sets doubts on the origin of the emission detected with the core LOS for which the recorded intensity was 1-2 orders of magnitude lower than for the SOL LOS, as shown by the decrease in the radial emission profiles in Figure 3c. This issue was investigated using the Xpaschen program [9] which calculates the expected shape of the spectral lines, taking into account the Zeeman splitting of the lines in a magnetic field. In Figures 3d-g, spectral lines measured on different toroidal LOS are compared to lines predicted by Xpaschen based on the magnetic conditions $13 \mathrm{~cm}$ above the HFS midplane without considering the Doppler shift and broadening of the lines. For the LOS closest to the inner wall (d) and near the separatrix (e), the calculated spectral lines agree well with the measured ones, whereas for the core LOS (f, g), the shapes of the measured and predicted lines are clearly different. This suggests that the LOS outside the separatrix observe the desired region in the HFS SOL, while 
the core LOS detect emission most probably from the LFS SOL at both ends of the LOS. Hence, the core measurements were excluded from the subsequent analysis.

\subsection{Determination of radial flow velocity and temperature profiles}

Radial flow velocity profiles were determined separately for $\mathrm{N}^{+}$and $\mathrm{N}^{2+}$ ions following a similar fitting procedure as in [3]. The N(II) and N(III) spectra were first generated by Xpaschen similarly as above without contributions of flow velocity and temperature, considering only the instrument function of the spectrometer. Doppler shift and broadening were then applied to the generated spectra by shifting and broadening the spectral lines according to different flow velocities and temperatures, until a match between the fitted and measured spectra was obtained. As presented in Figure 3a-b, the obtained fits reproduce the measured spectra accurately in the SOL in both toroidal (a) and poloidal (b) direction. The velocities and temperatures providing the best fit for each LOS then constitute the radial flow and temperature profiles.

The wavelength axes of the spectra were calibrated with a Ne lamp. Nominal and measured wavelengths were compared for two $\mathrm{Ne}$ (I) lines at a time, and the average of the resulting calibration shifts was used for the wavelength correction. Altogether four $\mathrm{Ne}(\mathrm{I})$ lines were observed close to the $\mathrm{N}$ lines used in the analysis, providing 10 different combinations for the wavelength calibration giving slightly different correction terms. This produces an uncertainty of approximately $3 \frac{\mathrm{km}}{\mathrm{s}}$ in the obtained flow velocities. The velocity profiles showed only minute differences below $1 \frac{\mathrm{km}}{\mathrm{s}}$ between different time instants. Therefore, the spectra were averaged over one second immediately after the maximal emission intensity was reached (see Figure $3 b$ ) to improve their signal-to-noise ratio, and the flow and velocity profiles were determined by fitting the averaged spectra. The profiles were found to be almost similar within $1 \frac{\mathrm{km}}{\mathrm{s}}$ also between different discharges, suggesting good repeatability of the measurements. An estimate for the radial uncertainty of the measurements was given by the spot sizes of $8 \mathrm{~mm}$ and $5 \mathrm{~mm}$ for the toroidal and poloidal LOS, respectively.

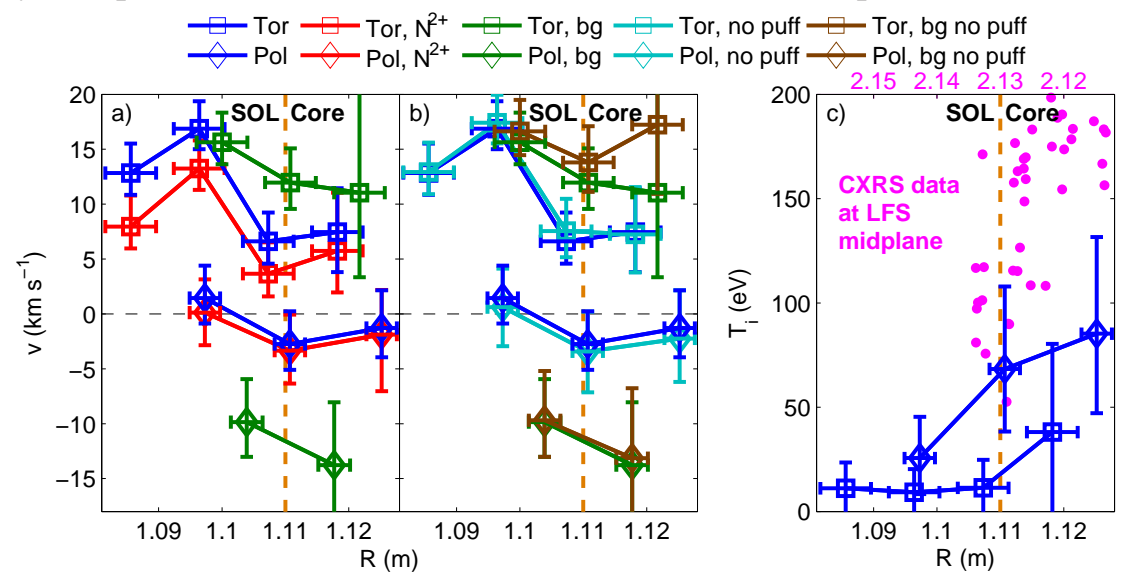

Figure 4: Flow velocity $(\mathrm{a}, \mathrm{b})$ and temperature $(\mathrm{c})$ profiles obtained in toroidal and poloidal direction for $\mathrm{N}^{+}$and $\mathrm{N}^{2+}$ ions. In plot (a), the flow profiles are presented for different ionization states and LOS, while the plot (b) shows comparison between the flows of injected and recycled $\mathrm{N}^{+}$ions. Unless otherwise noted, the legends refer to $\mathrm{N}^{+}$ions. The temperature results are compared to $T_{\mathrm{i}}$ data given by CXRS at the LFS midplane, corresponding to the magenta $R$ axis above the plot.

Our analysis suggests toroidal $\mathrm{N}$ flows of $5-20 \frac{\mathrm{km}}{\mathrm{s}}$ in co-current direction in the SOL. Poloidally, the $\mathrm{N}$ ions flow at approximately $3 \frac{\mathrm{km}}{\mathrm{s}}$ in the electron diamagnetic direction around the separatrix and at $1 \frac{\mathrm{km}}{\mathrm{s}}$ in the ion diamagnetic direction in the SOL. Figure $4 \mathrm{a}$ shows the obtained toroidal and 
poloidal flow velocity profiles in the SOL for both $\mathrm{N}^{+}$and $\mathrm{N}^{2+}$, as well as for $\mathrm{N}^{+}$measured with the toroidal and poloidal background LOS. The solid profiles are determined from time-averaged spectra, as described above, and are further averaged over different discharges and Ne calibration options. The uncertainties due to variations in time and between discharges and wavelength corrections have been taken cumulatively into account in the error bars.

The total flow is directed towards the lower HFS divertor at the separatrix and away from it in the near SOL. Since the toroidal flow is positive everywhere, the change of the direction of the poloidal flow is assumed to result from the perpendicular $\vec{E} \times \vec{B}$ drift rather than from transportdriven flow reversal. If the positive poloidal flow was assumed to be solely $\vec{E} \times \vec{B}$ drift, this would mean radial electric field of $E_{\mathrm{r}}<10 \frac{\mathrm{kV}}{\mathrm{m}}$ in the near SOL, which is of the same order as the values of $E_{\mathrm{r}}$ measured earlier in L mode in the edge plasma of ASDEX Upgrade [10]. A proper investigation of the drifts requires measurements in both forward and reversed field configurations.

For the $\mathrm{N}^{2+}$ ions, the toroidal and poloidal flow profiles are fairly similar to those of $\mathrm{N}^{+}$, although roughly $5 \frac{\mathrm{km}}{\mathrm{s}}$ slower flow is obtained in the toroidal direction. This is in contradiction with the observations made between $\mathrm{C}^{+}$and $\mathrm{C}^{2+}$ in [3], where a faster flow was measured for the $\mathrm{C}^{2+}$ ions which have a longer lifetime in the near SOL. However, the results for $\mathrm{N}^{+}$and $\mathrm{N}^{2+}$ agree within error bars, and the difference may be largely due to the $\mathrm{N}$ (III) lines being closely neighboured by significantly more intense N(II) lines, which complicates fitting of the N(III) lines.

Flow profiles were determined also for $\mathrm{N}^{+}$measured on the background LOS which detect emission of recycled $\mathrm{N}$ but may observe also part of the injected $\mathrm{N}$. In both directions - especially poloidally - the background LOS show faster flows than the signal LOS, suggesting different flows in different regions of the HFS SOL. However, as the background LOS do not directly observe the injection, the localization of these measurements cannot be guaranteed.

If only intrinsic recycled $\mathrm{N}^{+}$ions are considered by studying spectra measured before the injection, the resulting flow profiles are almost identical to the ones during the injection in both directions and for both signal and background LOS, as presented in Figure 4b. For the signal LOS, this is surprising, as the measurement is not expected to be localized to the HFS midplane without the injection but rather integrate the entire plasma along the LOS. The similarity of the profiles might thus suggest local enhancement of recycling of $\mathrm{N}$ deposited close to the injection site during the preceding discharges acting as a sufficiently strong source to localize the measurements also before the injection. The observation also shows that the velocity fit is reliable also for the weak and noisier signal in the absence of the injection. However, the signal was too weak for reliable fitting of the $\mathrm{N}(\mathrm{IIII})$ lines, and the flow profiles of $\mathrm{N}^{2+}$ could not be properly determined on the background LOS or before the injection.

Similar treatment was applied for the radial temperature profiles of the $\mathrm{N}^{+}$ions and their error bars as for the velocity profiles. An additional error of approximately $11 \mathrm{eV}$ was added due to an estimated 10-\% uncertainty of the instrument broadening of the spectrometer. Since the shape of the fitted spectral lines is more sensitive to decreased signal-to-noise ratio than their central wavelength, representative results were obtained only for the signal LOS during the injection. Due to the close proximity of the $\mathrm{N}(\mathrm{II})$ lines, reliable temperature fits were not produced for the N(III) lines.

The temperature profiles presented in Figure $4 \mathrm{c}$ show disagreement between the $\mathrm{N}^{+}$temperatures measured in toroidal and poloidal directions. While the toroidally measured temperature is rather constant at approximately $10 \mathrm{eV}$ throughout the SOL, the poloidal measurements show 
higher values increasing from $25 \mathrm{eV}$ to $70 \mathrm{eV}$ towards the separatrix. The reason for this mismatch is yet unclear. For comparison, $T_{\mathrm{i}}$ data measured by CXRS in the LFS pedestal region is presented, corresponding to the $R$ axis on top of the figure. Reliable measurements of $T_{\mathrm{i}}$ in the SOL do not exist, and definite conclusions on the equilibration of the $\mathrm{N}^{+}$ions with the background plasma cannot thus be made. However, based on the results with $\mathrm{C}^{+}$ions in [3], equilibrium is not expected. Measurements of $T_{\mathrm{i}}$ in the SOL, as well as modelling of the injection experiment, are required to fully address the equilibration of the $\mathrm{N}$ ions.

\section{Conclusions}

$\mathrm{N}$ was introduced in ASDEX Upgrade for measuring toroidal and poloidal plasma flows in the HFS SOL using Doppler spectroscopy. Measurements were done during several similar, lowdensity L-mode discharges by injecting $\mathrm{N}_{2}$ into the plasma near the HFS midplane and detecting the emission of singly and doubly ionized $\mathrm{N}$ by the HFS edge CXRS system.

The obtained radial flow profiles were found to be fairly invariant in time and similar for all discharges. The results suggest flow towards the HFS divertor close to the separatrix and away from it in the near SOL, most probably due to the $\vec{E} \times \vec{B}$ drift. Reasonable agreement was noticed between the flow profiles of $\mathrm{N}^{+}$and $\mathrm{N}^{2+}$ ions, and almost identical results were found for recycled and injected $\mathrm{N}^{+}$measured before and during the injection, respectively. The $\mathrm{N}^{+}$temperatures, obtained from the widths of the spectral lines, showed yet unexplained discrepancies between the toroidally and poloidally measured values. Equilibration of the $\mathrm{N}$ ions with the background plasma is not expected, but this could not be confirmed due to lack of reference data.

New experiments are planned for systematically investigating the behaviour of the impurity flows under varying SOL conditions in $\mathrm{L}$ and $\mathrm{H}$ mode plasmas. In addition, simulations will be carried out to study the equilibration of the $\mathrm{N}$ ions in different plasmas and to determine the background $\mathrm{D}$ flow for the measured $\mathrm{N}$ flows.

This work has been carried out within the framework of the EUROfusion Consortium and has received funding from the Euratom research and training programme 2014-2018 under grant agreement No 633053 [and from Tekes - the Finnish Funding Agency for Innovation under the FinnFusion Consortium]. The views and opinions expressed herein do not necessarily reflect those of the European Commission. We also acknowledge the contributions of the EUROfusion MST1 team.

\section{References}

[1] H.W. Müller et al., Deuterium plasma flow in the scrape-off layer of ASDEX Upgrade, Journal of Nuclear Materials 363-365 (2007) 605-610

[2] M. Tsalas et al., Divertor plasma flow near the lower x-point in ASDEX Upgrade, Plasma Physics and Controlled Fusion 49 (2007) 857-872

[3] T. Makkonen et al., Measurements and ERO simulations of carbon flows in the high-field side main SOL in AUG, Journal of Nuclear Materials 438 (2013) S410-S413

[4] T. Makkonen et al., Synthetic Doppler spectroscopy and curvilinear camera diagnostics in the ERO code, Computer Physics Communications 184 (2013) 1842-1847

[5] P. Heliste et al., Modelling impurity flows in the tokamak scrape-off layer with synthetic Doppler spectroscopy, Proceedings of the 49th Annual Conference of the Finnish Physical Society

[6] T. Pütterich et al., Poloidal asymmetry of parallel rotation measured in ASDEX Upgrade, Nuclear Fusion 52 (2012) 083013

[7] E. Viezzer et al., Rotation and density asymmetries in the presence of large poloidal impurity flows in the edge pedestal, Plasma Physics and Controlled Fusion 55 (2013) 124037

[8] L. Aho-Mantila et al., Outer divertor of ASDEX Upgrade in low-density L-mode discharges in forward and reversed magnetic field: II. Analysis of local impurity migration, Nuclear Fusion 52 (2012) 103007

[9] A. Pospieszczyk, Spectroscopic diagnostics of tokamak edge plasmas, Physica Scripta T119 (2005) $71-82$

[10] G.D. Conway et al., Impact of magnetic perturbation coils on the edge radial electric field and turbulence in ASDEX Upgrade, Plasma Physics and Controlled Fusion 57 (2015) 014035 